\title{
Panbiogeografía, componentes bióticos y zonas de transición
}

\author{
Juan J. Morrone ${ }^{1}$
}

'Museo de Zoología 'Alfonso L. Herrera', Departamento de Biología Evolutiva, Facultad de Ciencias, Universidad Nacional Autonoma de México. Apdo. postal 70-399, 04510 México, D.F., México.

\begin{abstract}
Recognition of biotic components is a key element for understanding evolution in space-time. Panbiogeography represents a biogeographic approach that may be applied to identify these biotic components. A revision of the panbiogeographic approach is provided herein, including definitions of basic concepts (individual and generalized tracks, nodes, baselines, and main massings) and methods (manual, connectivity and incidence matrices, track compatibility, and parsimony analysis of endemicity). As an example of this approach, 70 biotic components of Latin America and the Caribbean are identified, briefly characterized, and arranged in a biogeographic system of three regions, seven subregions, and two transition zones. The relevance of the recognition of biotic components and transition zones is discussed.
\end{abstract}

KEYwORDs. Biogeography; evolution; Neotropics; nodes; parsimony analysis of endemicity; tracks.

Resumen. El reconocimiento de componentes bióticos resulta clave para comprender la evolución en espacio-tiempo. La panbiogeografía representa un enfoque biogeográfico que puede aplicarse para identificar estos componentes bióticos. Se proprorciona una revisión del enfoque panbiogeográfico, incluyendo definiciones de conceptos básicos (trazos individuales y generalizados, nodos, líneas de base y centros de masa) y métodos (manual, matrices de conectividad e incidencia, compatibilidad de trazos y análisis de parsimonia de endemismos). Como un ejemplo de este enfoque, se identifican 70 componentes bióticos de América Latina y el Caribe, se caracterizan brevemente, y se ordenan en un sistema biogeográfico de tres regiones, siete subregiones y dos zonas de transición. Se discute la relevancia del reconocimiento de componentes bióticos y zonas de transición.

KeYwords. Biogeografía; evolución; Neotrópicos; nodos; análisis de parsimonia de endemismos; trazos.

La biogeografía estudia la distribución de los seres vivos en espacio y tiempo, al reconocer patrones de distribución, proponer hipótesis acerca de los procesos que los causaron y proporcionar un sistema de regionalización biótica del planeta. Esta disciplina es idiosincrásica, pues pese a la existencia de numerosos libros, simposios y cursos sobre la misma, raramente hay "biogeógrafos" (NELSON 1985), lo cual se debería a que ocupa un espacio intermedio entre la geografía, la geología y la biología, siendo practicada por sistemáticos, ecólogos, paleontólogos y geógrafos, entre otros. En la actualidad, la biogeografía atraviesa por un periodo extraordinario de renovación teórica y metodológica (MORRONE \& CRisci 1995; ANDERSSON 1996; VuILleumier 1999; CRisci 2001).

Durante la historia de la biogeografía se han desarrollado varios enfoques diferentes, los que pueden clasificarse en dos grandes campos, denominados biogeografía ecológica y biogeografía histórica. La biogeografía ecológica generalmente analiza patrones de distribución individual o poblacional, a escalas espaciales y temporales pequeñas, mientras que la biogeografía histórica básicamente analiza patrones de distribución de especies y taxones supraespecíficos, a escalas espaciales y temporales mayores (MORRONE et al.1996; VARGAS 2002). Esta distinción es artificial, pues implica dividir un continuo, donde los extremos son identificables fácilmente como "ecológico" o "histórico", pero en la parte media resulta difícil justificar tal distinción, como ocurre con algunos enfoques, como la biogeografía de los refugios cuaternarios, la macroecología o la filogeografía. Esta dicotomía historia/ ecología es semejante a otras dicotomías que oponen factores internos y externos, planteadas en la biología e incluso fuera del ámbito biológico (Oyama 2000). En el caso de la biogeografía, varios autores han criticado la falta de integración entre la biogeografía histórica y la ecológica, llegando a sostener que sería deseable integrarlas en un programa de investigación unificado (Gray 1989; SMith 1989; Henderson 1991;VARGAS 1992a; Morrone 1993a; Zunino \& Zullini 1995; HollowaY 2003). HAYDON et al. (1994) conceptualizaron a la biogeografía a partir de una estructura triangular, donde los patrones biogeográficos resultan de la interacción entre procesos ecológicos, históricos y estocásticos, lo cual parece un buen punto de partida para una futura síntesis.

Dentro del campo de lo que se conoce como biogeografía histórica se ha desarrollado un enfoque conocido como panbiogeografía (CroizAT 1958, 1964; Craw et al. 1999). Surgida como reacción al dispersalismo de DARWIN (1859) y WALLACE (1876) - enfoque que presupone que las especies se originan en centros de origen, a partir de los cuales se dispersan al azar, atraviesan barreras preexistentes y colonizan nuevas áreas la panbiogeografía se constituyó durante las últimas décadas del siglo pasado en un programa de investigación vigoroso (Craw \& Weston 1984). Hace una década y media, Morrone \& CRISCI (1990) llevaron a cabo una revisión en español de los principios teóricos y metodológicos de la panbiogeografía. Luego de dicha revisión, ha aparecido una obra de síntesis (CRAw et al. 1999), se han producido algunos avances metodológicos - sobre todo la implementación del análisis de 
parsimonia de endemismos para llevar a cabo análisis panbiogeográficos - y se han publicado numerosos análisis aplicando principios panbiogeográficos (ver Cuadro I). Por ello, una revisión actualizada de los principios y métodos de la panbiogeografía me parece apropiada.

Mis objetivos son discutir el marco teórico donde se sitúa la panbiogeografía, introducir sus conceptos básicos y metodologías principales, y presentar brevemente un sistema biogeográfico de América Latina y el Caribe, donde se aplican los principios panbiogeográficos para reconocer componentes bióticos y ordenarlos jerárquicamente.

\section{MARCOTEÓRICO}

¿Qué es la panbiogeografía? Resulta difícil caracterizar a la panbiogeografía teniendo en cuenta la diversidad de opiniones existentes. Para PATterson (1981) es "fenética", pues se basaría en similitud global. Para MAYR (1982) es "excéntrica". Nelson (1989) la considera como una "metateoría evolutiva". Stace (1989) la define como "vicarianza en patrones de distribución globales". Grene (1990) la considera "caprichosa". Para algunos autores es una precursora de la biogeografía cladística (Nelson \& Platnick 1981; Grande 1990; Cox 1998). Para otros es un programa de investigación alternativo (CRAW \& Weston 1984; Humphries \& Seberg 1989; Platnick \& Nelson 1988; Morrone \& Crisci 1990, 1995; Vargas 1992b; Zunino \& Zullini 1995; Morrone et al. 1996; ColaCino 1997; CrisCi et al. 2000; Grehan 2001d). Finalmente, hay quienes proponen que la panbiogeografía posee implicancias importantes para los estudios evolutivos (GreHAN 1984, 1988a, 2001b; GreHAN \& Ainsworth 1985; Heads 1985; Gray 1988, 1992; Morrone 2000c).

De acuerdo con CRAW et al. (1999), la panbiogeografía enfatiza la dimensión espacial o geográfica de la biodiversidad, para permitirnos una mejor comprensión de los patrones y procesos evolutivos. La comprensión de la dimensión espacial de los seres vivos, a partir del análisis de sus distribuciones geográficas, es un prerrequisito para los estudios evolutivos, ya que la geografía es el sustrato sobre el cual tiene lugar la historia de la vida. Una manera menos "pretenciosa" de ver a la panbiogeografía es considerarla un método que permite llevar a cabo una exploración inicial de los datos, antes de llevar a cabo un análisis biogeográfico cladístico.

Vicarianza versus dispersión. Uno de los debates más importantes en la historia de la biogeografía de los siglos XIX y XX se refirió a cuál proceso biogeográfico histórico es más importante, la dispersión o la vicarianza (NELSON 1978; PLATNICK \& Nelson 1978; Nelson \& Platnick 1981; Humphries \& Parenti 1999). Clásicamente, se consideró que la dispersión era el factor más importante. A partir de las ideas de DARWIN (1859) y WALLACE (1876) se desarrolló la biogeografía dispersalista, que enfatizó la dispersión a partir de centros de origen para explicar las distribuciones de los seres vivos (ver MatTHEW 1915 y Simpson 1965). A mediados del siglo XX, la vicarianza surgió como una explicación alternativa a la dispersión (CROIZAT
1964; CROIZAT et al. 1974). Un siglo antes, sin embargo, HOOKER (1844-60) había descubierto que, paradójicamente, tanto la dispersión como la vicarianza podían emplearse para explicar las mismas disyunciones. Por ejemplo, podemos tener una especie que habita dos áreas, lo cual puede deberse a que se encontraba distribuida ampliamente cuando ambas se encontraban unidas - explicación por vicarianza - o que se originó en alguna de ellas y de allí se dispersó a la otra explicación por dispersión (MORRONE 2002).

Los biogeógrafos del siglo XIX prefirieron a la dispersión sobre una geografía estable como el mecanismo primario, aplicando el enfoque dispersalista, que imperaría hasta mediados del siglo XX. Sin embargo, la solución de la paradoja de Hooker ( $l$. c.) no consiste en elegir un mecanismo u otro, sino que hay que desarrollar un razonamiento diferente (GrehAN 1991). En realidad, el proceso de vicarianza también incluye a la dispersión, aunque ésta ocurre antes de que surjan las barreras geográficas. De acuerdo con esta concepción, las distribuciones geográficas de las especies evolucionan en dos etapas:

1. Cuando los factores climáticos y geográficos son favorables, los organismos están en estado de "movilidad", por lo que expanden activamente su área de distribución geográfica de acuerdo con sus capacidades de dispersión o vagilidad, adquiriendo las especies su distribución ancestral o cosmopolitismo primitivo. Esta etapa corresponde al proceso de dispersión.

2. Cuando los organismos han ocupado todo el espacio geográfico o ecológico disponible, su distribución se estabiliza. Este periodo de "inmovilidad" permite el aislamiento espacial de las poblaciones en distintos sectores del área, mediante el surgimiento de barreras geográficas y la consecuente diferenciación de nuevas especies. Esta etapa corresponde al proceso de vicarianza.

Homología biogeográfica. Homología significa "equivalencia de partes", siendo el procedimiento empleado para establecer comparaciones taxonómicas válidas (DE PINNA 1991). Se trata de un concepto comparativo, donde cada enunciado de homología individual interactúa con otros enunciados semejantes, por ejemplo, cuando aseguramos que las alas de las aves son homólogas de las patas anteriores de los mamíferos, pero no lo son de las alas de los insectos. Partiendo de la analogía entre taxonomía y biogeografía, podemos considerar las distribuciones de taxones individuales como los enunciados sobre homología biogeográfica que comparamos (MORRONe 2001g), reconociendo los mismos estadios que DE PINNA (1991) propuso para la sistemática: la homología primaria (estadio de generación de hipótesis) es una conjetura sobre la correspondencia entre partes de organismos diferentes, y la secundaria (estadio de legitimación de hipótesis) representa la contrastación de dicha conjetura, a través de la congruencia con enunciados similares en el cladograma.

La homología biogeográfica primaria se refiere a una conjetura sobre una historia biogeográfica común, la cual 
postula que diferentes taxones - aun teniendo medios de dispersión diferentes - se hallan integrados espaciotemporalmente en un mismo componente biótico (MORRONE 2001g). Cabe destacar que varios autores han trabajado previamente con conceptos semejantes al de componente biótico (Jeannel 1942; Croizat 1958, 1964; Ringuelet 1961; Reig 1962, 1981; Baroni-Urbani et al. 1978; HalfFTER 1978, 1987, 2003; CADLE 1985). Un modo de postular hipótesis de homología biogeográfica primaria es a partir de un análisis panbiogeográfico, el cual compara trazos individuales de taxones diferentes para detectar trazos generalizados o componentes bióticos.

La homología biogeográfica secundaria se refiere a la contrastación de la homología primaria hipotetizada en la etapa anterior (MORRONE 2001g). Un análisis biogeográfico cladístico nos permite comparar cladogramas taxonómicos de áreas obtenidos remplazando en los cladogramas taxonómicos los taxones terminales por los componentes bióticos que ellos integran - para obtener un cladograma general de áreas (Morrone \& Carpenter 1994; Morrone \& Crisci 1995; Humphries \& PARENTI 1999; CRISCi et al. 2000).

De acuerdo con estas consideraciones sobre homología biogeográfica, la disputa entre panbiogeógrafos y biogeógrafos cladistas acerca de la superioridad de alguno de los enfoques partiría de una concepción errónea: que ambos poseen el mismo objetivo. De hecho, la panbiogeografía trata con la homología biogeográfica primaria, mientras que la biogeografía cladística trata con la secundaria, por lo que sería posible integrarlas como etapas sucesivas de un mismo análisis (Morrone \& Crisci 1990, 1995; Crisci et al. 2000; Donoghue et al. 2001; Morrone 2001g).

\section{CONCEPTOS BÁSICOS}

Trazos individuales. Un trazo individual es la unidad básica de un estudio panbiogeográfico, constituyendo las coordenadas primarias de un taxón en el espacio. Operativamente, consiste en una línea que conecta las localidades donde se distribuye una especie o taxón supraespecífico, de modo que la suma de los segmentos que conectan las localidades sea la menor posible. Desde el punto de vista topológico, un trazo individual es un árbol de tendido mínimo, que para $\mathrm{n}$ localidades contiene $\mathrm{n}-1$ conexiones (PAGE 1987). Cuando se hace un trazo para conectar las distintas localidades donde se distribuye una especie, el criterio a seguir es relativamente sencillo. Se encuentran las dos localidades más cercanas y se conectan por medio de una línea; luego, este par de localidades se conecta con la localidad más cercana a cualquiera de las dos. Después se une la localidad más cercana a cualquiera de las tres, y así sucesivamente (Figs. 1-6). El resultado es un árbol lineal o ramificado, no enraizado, en que la suma de los segmentos que conectan las localidades es mínima, siguiendo una especie de "parsimonia geográfica". Los trazos individuales interpretan la geometría espacial como un componente explícito, difiriendo así de los mapas de distribución que engloban las localidades de un taxón mediante una línea (GREHAN 2001b).

La orientación de los trazos individuales representa la formulación de una hipótesis sobre la secuencia de las disyunciones implicadas en él. La manera más frecuente de orientar un trazo es a partir de la designación de una línea de base (Fig. 7), la cual representa un rasgo diagnóstico primario de un trazo individual, que puede interpretarse como el sitio de localización de la biota ancestral (Grehan 1991). Para la designación de la línea de base, se deben analizar los rasgos geográficos/ geológicos de mayor relevancia, que signifiquen división de áreas geológicas, climáticas o de continuidad de hábitat. A escala global, los rasgos geológicos más relevantes que pueden usarse como líneas de base son las grandes cuencas oceánicas o marinas. A escalas menores, algunos accidentes geológicos evidentes son brazos de mar, cadenas montañosas y ríos. Si se trata de orientar un trazo estrictamente terrestre, la situación es más complicada, ya que a veces se debe decidir si la disyunción debida, por ejemplo, a la interposición de una cordillera, es más antigua que la debida a la interposición de un río u otro accidente geológico. Resulta inexplicable que GrehAN (1994) haya considerado que las líneas de base equivalen a "centros de origen". Aunque este autor aclaró que no se trata del mismo concepto del dispersalismo, creo que esta equivalencia es errónea y podría dar lugar a confusión.

Para orientar el trazo individual de una taxón supraespecífico, se puede emplear un criterio filogenético (Fig. 8). La interpretación de este criterio en el trabajo de Croizat se circunscribió al hecho que los trazos conectaban las áreas o localidades del mismo grupo taxonómico. PAGE (1987) sugirió la posibilidad de usar la información cladística para la orientación de los trazos individuales (Fig. 8). Esta sugerencia luego fue aceptada por Craw (1988), Henderson (1989) y Grehan (1991). Sin embargo, Platnick \& Nelson (1988) advirtieron que la aplicación de este criterio puede caer en un uso análogo al de la biogeografía filogenética de Hennig, lo cual sería criticable aun por los mismos panbiogeógrafos. Por otro lado, si consideramos a los resultados de los análisis panbiogeográficos como hipótesis de homología biogeográfica primaria, las cuales contrastaremos luego mediante un análisis biogeográfico cladístico, resulta problemático orientar los trazos mediante información filogenética, porque ésta intervendría tanto en la formulación de las hipótesis (análisis panbiogeográfico) como en su contrastación ulterior (análisis biogeográfico cladístico), cayéndose en un razonamiento de tipo circular.

Otro criterio para la orientación de los trazos individuales es la localización de los centros de masa (Fig. 9), definidos como los núcleos de mayor riqueza de especies dentro de la distribución de un taxón superior. En general, los centros de masa representan áreas de diversidad numérica, genética o morfológica de un grupo (Page 1987). Platnick \& Nelson (1988) criticaron el concepto de centro de masa, pues consideraron que los mismos también fueron referidos por Croizat como "centros de dispersión", "lugares de origen", "centros de emergencia", "centros ancestrales de radiación" y aun 
Cuadro I. Métodos para identificar componentes bióticos bajo el enfoque panbiogeográfico.

\begin{tabular}{ll}
\hline Métodos & Aplicaciones empíricas \\
\hline Reconstrucción manual & Croizat (1958, 1964), Rosen (1976), Heads (1986, 1989), Grehan (1988b, 2001a-c), Morrone \\
& $(1993 b, 1994 a, 1996$, 2000a, b, 2001a-f), Franco Rosselli \& Berg (1997), Lopretto \& \\
& Morrone (1998), Lourenço (1998), Contreras Medina et al. (1999), Katinas et al. (1999), \\
& Morrone \& Pereira (1999), Menu Marque et al. (2000), Luna Vega \& Contreras Medina \\
& (2000), Aguilar Aguilar \& Contreras Medina (2001), Contreras Medina \& Eliosa León \\
& (2001), Carvalho et al. (2003) y Roig Juñent et al. (2003).
\end{tabular}

Matrices de conectividad e incidencia Page(1987)

Compatibilidad de trazos

Craw (1988, 1989a), Morrone (1992), Morrone \& Lopretto (1994), Posadas et al. (1997) y Crisci et al. (2001).

Análisis de parsimonia de Rosen(1988), Cracraft (1991), Myers (1991), Morrone (1994b, 1998), Fernandes et al. endemismos (1995), Morrone \& Lopretto (1995), Da Silva \& Oren (1996), Morrone \& Coscarón (1996), Posadas (1996), Bellan \& Bellan Santini (1997), Cortés \& Franco (1997), Morrone et al. (1997, 1999), Posadas et al. (1997), Geraads (1998), Linder \& Mann (1998), Sfenthourakis \& Giokas (1998), Watanabe (1998), Glasby \& Álvarez (1999), Luna Vega et al. (1999, 2000, 2001), Espinosa Organista et al. (2000), Ron (2000), Bisconti et al. (2001), Crisci et al. (2001), Ippi \& Flores (2001), Marino et al. (2001), Morrone \& Márquez (2001), Trejo Torres \& Ackerman (2001, 2002), García Barros et al. (2002) y Rojas Soto et al. (2003).

“centros de origen". Hasta uno de los autores que inicialmente apoyó la panbiogeografía tuvo que reconocer que el concepto de centro de masas es "horriblemente vago" (PAGE 1990). Si un trazo se orienta a partir del centro de masa hacia la periferia, la inferencia involucrada en esa hipótesis sería semejante a la de la biogeografía dispersalista (CRISCI et al. 2000), por lo que la orientación con este criterio sería desaconsejable.

En síntesis, de los criterios que pueden usarse para la orientación, el menos problemático sería el de la línea de base. Sin embargo, éste solo es aplicable en ciertas condiciones, ya que cuando el análisis panbiogeográfico se realiza a escala continental, el empleo de rasgos geológicos o tectónicos resulta difícil de llevar a cabo. Por ello, creo que lo más aconsejable es llevar a cabo los análisis panbiogeográficos a partir de trazos individuales no orientados, como de hecho ocurre en la mayor parte de los análisis publicados.

Trazos generalizados. Los trazos generalizados o estándar resultan de la superposición estadísticamente significativa de trazos individuales que conectan áreas de distribución (ZUNINO \& ZULLINI 1995). Representan patrones de distribución actuales de biotas ancestrales, las cuales fueron fragmentadas por eventos geológicos o tectónicos (CRAW 1988). Los trazos generalizados resultan luego de un análisis comparativo de los trazos individuales, en el que se evalúa la congruencia en su topología (Fig. 10). Cuando los trazos individuales que se comparan poseen orientación, se considera que integran un mismo trazo generalizado cuando coinciden tanto en su estructura como en su dirección (CRAw 1988).

Los trazos generalizados equivalen a componentes bióticos (Morrone 2001g), los cuales pueden ser ordenados jerárquicamente en un sistema de clasificación biogeográfica. Si bien esencialmente resultan de vicarianza, el proceso de dispersión también ha contribuido a moldearlos. Sin embargo, creo que más que intentar interpretarlos en relación con esto procesos, deberían ser considerados como enunciados de homología biogeográfica primaria.

Nodos. Los nodos son áreas complejas, donde dos o más trazos generalizados se superponen (Fig. 10). Fueron interpretadas por Croizat como zonas de convergencia geobiótica. El reconocimiento de nodos es uno de los aportes más importantes de la panbiogeografía, y constituye el punto de partida para especular sobre la existencia de áreas compuestas. Croizat y sus seguidores han basado muchas de sus críticas a la biogeografía cladística en la incapacidad de ésta para distinguir este tipo de áreas compuestas y complejas, debido a su apego a la jerarquía implícita en los cladogramas. Esto no sería del todo correcto, ya que las áreas compuestas se deberían comportar en el cladograma general de áreas como especies de origen híbrido, mostrando conflicto en sus relaciones con áreas "paternales" diferentes.

CRAW et al. (1999) y GREHAN (2001b) denominaron también nodos a las localidades de intersección de trazos individuales. Los nodos hallados en la intersección de los trazos generalizados, entonces, deberían denominarse "nodos 


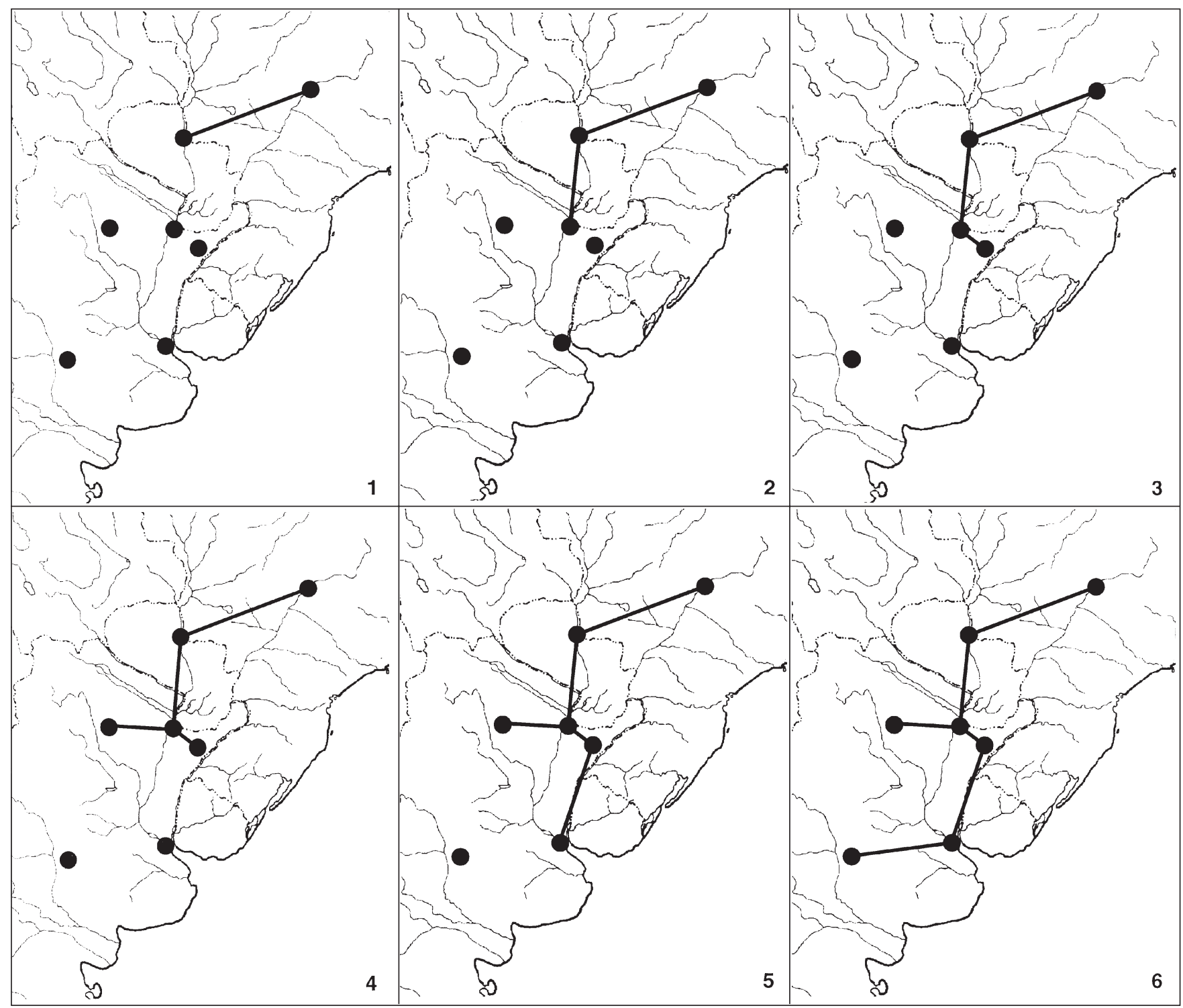

Figs. 1-6. Obtención de un trazo individual, uniendo entre sí las localidades de distribución de una especie, de modo que cada una se conecte con la más cercana.

generalizados".

\section{MÉTODOS}

Existen diferentes aproximaciones metodológicas (Cuadro I) para llevar a cabo análisis panbiogeográficos (CROIZAT 1958, 1964; Page 1987; Craw 1988; Morrone \& Crisci 1990, 1995; Craw et al. 1999; Crisci et al. 2000; VARGAS 2002).

Método manual. El método propuesto originalmente por CroizAt $(1958,1964)$ consiste simplemente en delinear en mapas los trazos individuales de distintos taxones y luego superponerlos con el fin de determinar trazos generalizados.

El método básicamente comprende los siguientes pasos: 1) construir trazos individuales para taxones diferentes, conectando las localidades donde se distribuyen mediante un árbol de tendido mínimo; 2) de ser posible, orientar los trazos individuales a partir de líneas de base; 3) reconocer trazos individuales semejantes (en los trazos orientados, si poseen la misma dirección), a los que se considerará como parte de un mismo trazo generalizado; 4) reconocer nodos en las áreas donde dos o más trazos generalizados se superpongan; 5) indicar en un mapa los trazos generalizados, las líneas de base y los nodos.

Matrices de conectividad e incidencia. El método propuesto por PAGE (1987) se fundamenta en la teoría de grafos. De acuerdo con este método, el análisis de trazos equivale al diseño de un árbol de tendido mínimo, que conecta todas las localidades de un taxón de modo que la suma de las distancias sea mínima 


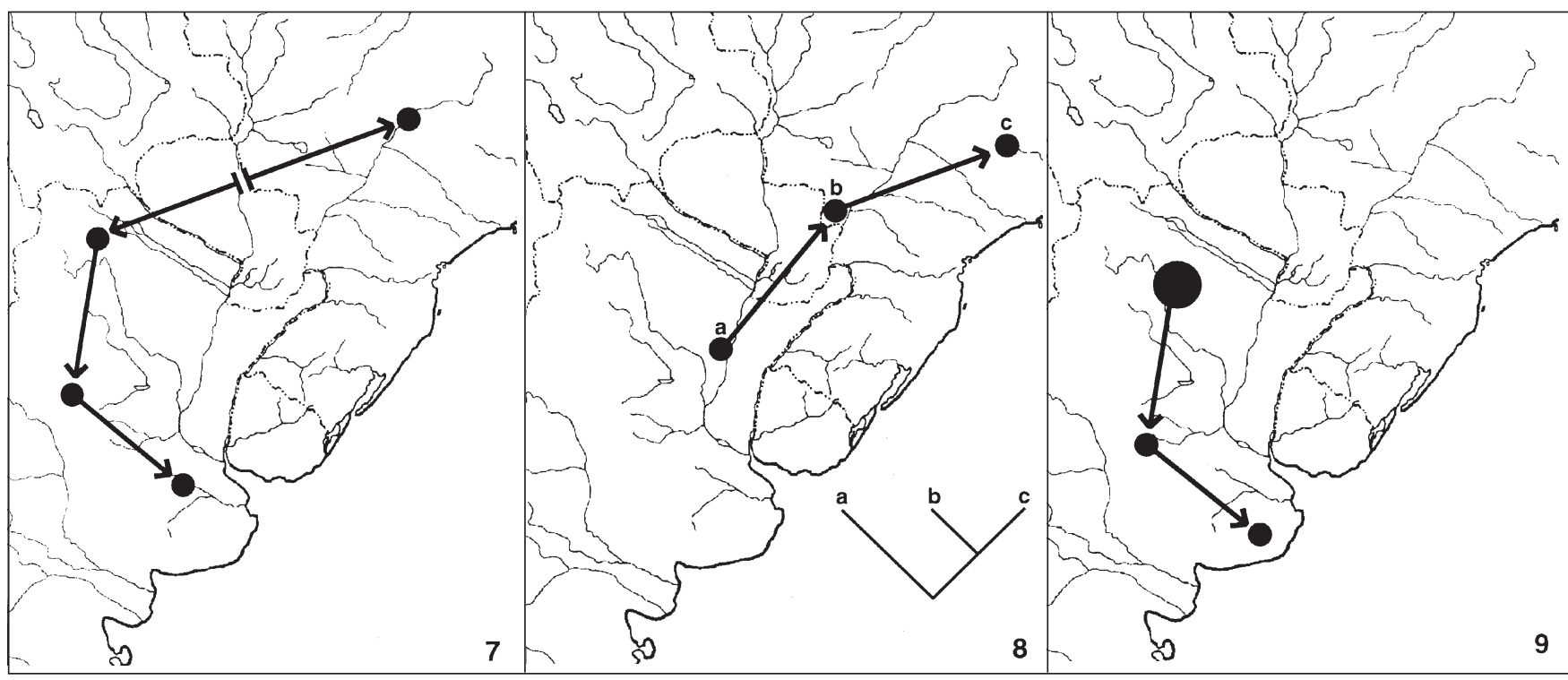

Figs. 7-9. Orientación de trazos individuales: 7, a partir de una línea de base; 8, empleando información filogenética; 9, a partir de un centro de masa.

(PAGe 1987; Henderson 1989; Vargas 2002). Una vez delineados en el mapa los trazos individuales, se vuelca la información que contienen en matrices de conectividad y de incidencia. Las matrices de conectividad son matrices $\mathrm{n} \mathrm{x}$, donde " $\mathrm{n}$ " es el número de puntos (localidades o áreas de distribución). Las matrices de incidencia son matrices i $\mathrm{x}$ j, donde "i $\mathrm{i}$ " es el número de puntos (localidades o áreas) y “j” el número de segmentos que conectan dichos puntos.

El método comprende los siguientes pasos (PAGE 1987; Craw \& Page 1988; Craw 1989a; Morrone \& Crisci 1990, 1995; Morrone et al. 1996): 1) construir trazos individuales para taxones diferentes, conectando las localidades donde se distribuyen mediante un árbol de tendido mínimo; 2) orientar los trazos individuales a partir de líneas de base; 3) construir matrices de conectividad para los trazos individuales y una matriz de conectividad global para todos ellos reunidos, para evaluar la congruencia entre los trazos, considerando que las localidades con índices de conectividad altos representan nodos; 4) construir matrices de incidencia, para reconocer trazos individuales orientados en la misma dirección, los que se considera que integran un mismo trazo generalizado; 5) indicar en un mapa los trazos generalizados, las líneas de base y los nodos.

Compatibilidad de trazos. CRAW $(1988,1989$ a) formalizó un método cuantitativo, con base en la compatibilidad de caracteres. El análisis de compatibilidad de caracteres considera como caracteres compatibles a aquellos que cuando se comparan entre sí no se contradicen en cuanto a los grupos que sustentan. En su implementación panbiogeográfica, el concepto de compatibilidad es más estricto que en sistemática, dado que dos o más trazos son compatibles solo si son iguales o uno se sitúa dentro del otro (CRISCI et al. 2000). Al conjunto de caracteres compatibles entre sí se lo llama "clique"; éste se emplea para construir el trazo generalizado que conecta las áreas, trazándoselo sobre un mapa. Para permitir la evaluación estadística del trazo generalizado, se generan al azar matrices del mismo tamaño que la matriz real; cuanto mayor sea el número de matrices generadas al azar que presenten "cliques" iguales o mayores que los obtenidos a partir de los datos reales, la significación estadística del trazo generalizado será menor.

El algoritmo comprende los siguientes pasos (CRAW 1989a; Morrone \& Crisci 1995; Morrone et al. 1996; Crisci et al. 2000; GREHAN 2001b): 1) construir trazos individuales para taxones diferentes, conectando las localidades donde se distribuyen mediante un árbol de tendido mínimo; 2) construir una matriz de áreas por trazos individuales, en la que la presencia se representa con un "1" y la ausencia con un " 0 "; 3 ) hallar el "clique" de trazos individuales compatibles mayor, que será considerado como un trazo generalizado; 4) identificar las líneas de base; 5) indicar en un mapa los trazos generalizados, las líneas de base y los nodos.

Análisis de parsimonia de endemismos. El análisis de parsimonia de endemismos permite construir cladogramas a partir de matrices de presencia-ausencia de especies y taxones supraespecíficos (Rosen 1988; CRACRAFT 1991; Myers 1991; Morrone 1994b, c; Escalante \& Morrone 2003; Trejo Torres 2003). Algunos autores han propuesto que el análisis de parsimonia de endemismos puede utilizarse para llevar a cabo análisis panbiogeográficos, donde los clados obtenidos son considerados como trazos generalizados (CRAW et al. 1999; Luna Vega et al. 2000; Morrone \& Márquez 2001). Con el objeto que los caracteres derivados aparezcan una única vez y no reviertan a " 0 " (de modo semejante a un análisis de 
compatibilidad), Luna Vega et al. (1999) llevaron a cabo el análisis de parsimonia con la concavidad de Goloboff $\mathrm{k}=0$ con el programa PAUP 4.0.1 (Swofford 1999). Luna Vega et al. (1999) y GARCÍA BARRos et al. (2002) propusieron que una vez que se han obtenido los cladogramas más parsimoniosos, es posible "desconectar" las especies que sustentan los diferentes clados y analizar nuevamente la matriz para buscar clados sustentados por otros taxones.

El algoritmo comprende los siguientes pasos (CRAW 1989b; Morrone 1994c; Posadas \& Miranda-Esquivel 1999; Crisci et al. 2000; GrehAN 2001b; VARGAS 2002): 1) construir trazos individuales para taxones diferentes, conectando las localidades donde se distribuyen mediante un árbol de tendido mínimo; 2) construir una matriz de áreas por trazos individuales, en la que la presencia se representa con un "1" y la ausencia con un "0", añadiéndose un área "externa" con todos " 0 " con el objeto de enraizar el cladograma; 3 ) analizar la matriz con un algoritmo de parsimonia y conectar las áreas incluidas en cada clado sustentado por al menos dos trazos individuales como parte de un mismo trazo generalizado; 4) "desconectar" los trazos individuales que sustentan los clados obtenidos y analizar nuevamente la matriz para buscar clados sustentados por otros; 5) indicar en un mapa los trazos generalizados y nodos.

\section{COMPONENTES BIÓTICOS DE AMÉRICA LATINA YELCARIBE}

Con el objeto de ejemplificar la aplicación del enfoque panbiogeográfico, presento una síntesis de varios análisis previos (ver MORRONE 2001a), a partir de los cuales reconocí 70 componentes bióticos en América Latina y el Caribe, a los cuales di categoría de provincia biogeográfica (Fig. 11). Éstos a su vez se agruparon en componentes bióticos mayores, considerados como regiones, subregiones y dominios. Además, he reconocido dos zonas de transición (Fig. 12). A continuación detallo brevemente las provincias biogeográficas de América Latina y el Caribe, junto con las regiones, subregiones, dominios y zonas de transición en que éstas se agrupan.

Región Neártica. Básicamente corresponde a las áreas templado-frías de América del Norte, en Canadá, los Estados Unidos (excluyendo el sur de la Florida) y el norte de México. Se asigna al reino Holártico, el cual también incluye la región Paleártica (Europa, Asia al norte del Himalaya, África al norte del Sahara y Groenlandia), y corresponde al paleocontinente de Laurasia. Las provincias mexicanas de la región Neártica se asignan a la subregión Pacífica Norteamericana (MORRONE et al. 1999), agrupándose en dos dominios. El dominio Californiano comprende dos provincias: California (norte de la península de Baja California, desde las montañas de San Pedro Mártir y Juárez, extendiéndose a lo largo de la Sierra Nevada hasta el sudoeste de los Estados Unidos) y Baja California (península de Baja California). El dominio Neártico Continental comprende tres provincias: Sonora (áreas costeras

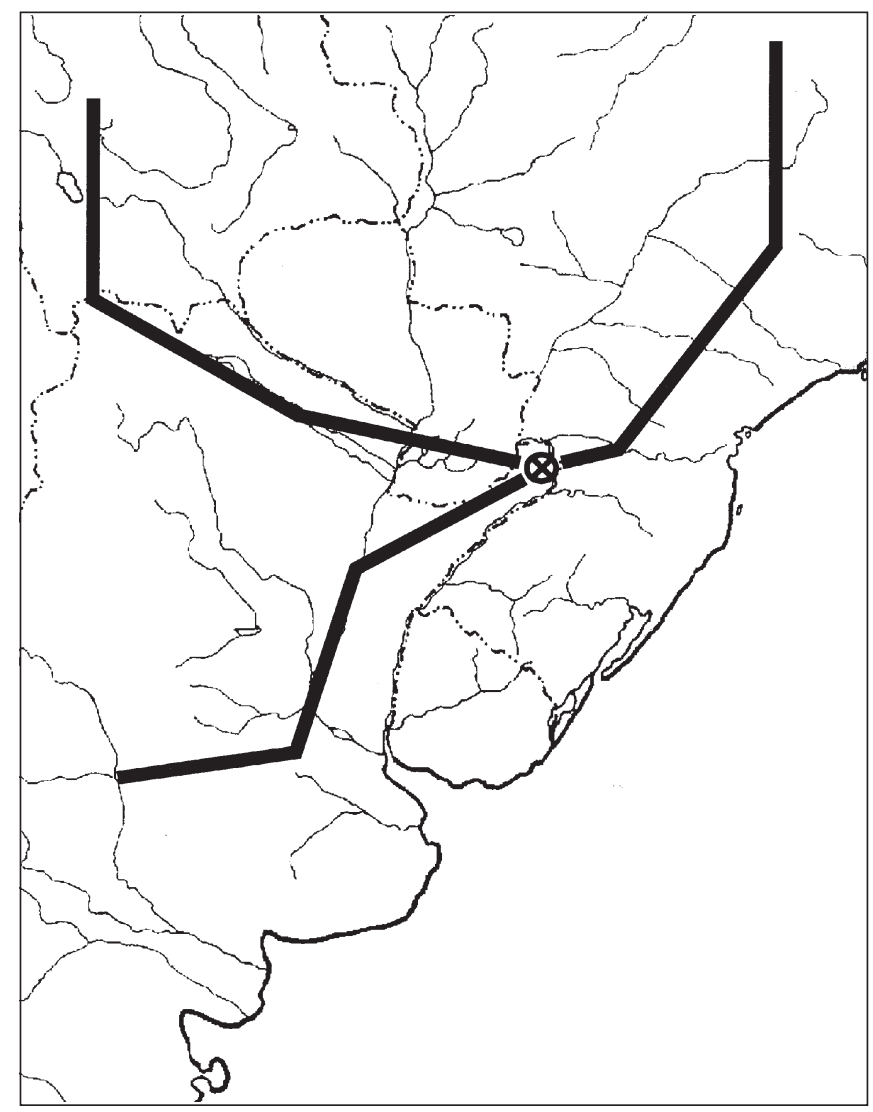

Fig. 10. Tres trazos generalizados, que se superponen en un nodo.

del noroeste de México, desde la porción nororiental de la península de Baja California hasta el río Piaxtla en el sur), Altiplano Mexicano (altiplano del centro de México, situado entre las Sierras Madre Occidental y Oriental) y Tamaulipas (áreas costeras del norte del Golfo de México, al norte del río Pánuco).

Zona de transición Mexicana. Halffter $(1978,1987,2003)$ definió esta zona como el área donde se superponen elementos neárticos y neotropicales en el sudoeste de los Estados Unidos, México y la mayor parte de América Central. Halffter analizó los patrones de distribución de Scarabaeidae (Coleoptera) de montaña, estableciendo tres patrones de distribución. El patrón Mesoamericano incluye taxones que se diversificaron en el oligoceno en hábitats montanos húmedos, cuya mayor diversidad se encuentra en América Central y sus afinidades son sudamericanas. El patrón Paleoamericano incluye taxones que se diversificaron en el plioceno, antes del cierre del istmo de Tehuantepec, los cuales se restringen a áreas montañosas de México, con preferencia por desiertos, pastizales y selvas lluviosas; pueden poseer algunas especies en América Central; y sus afinidades son con taxones del Viejo Mundo. El patrón Neártico incluye taxones que se diversificaron en las montañas de México durante el plioceno y pleistoceno, constituyendo el istmo de 


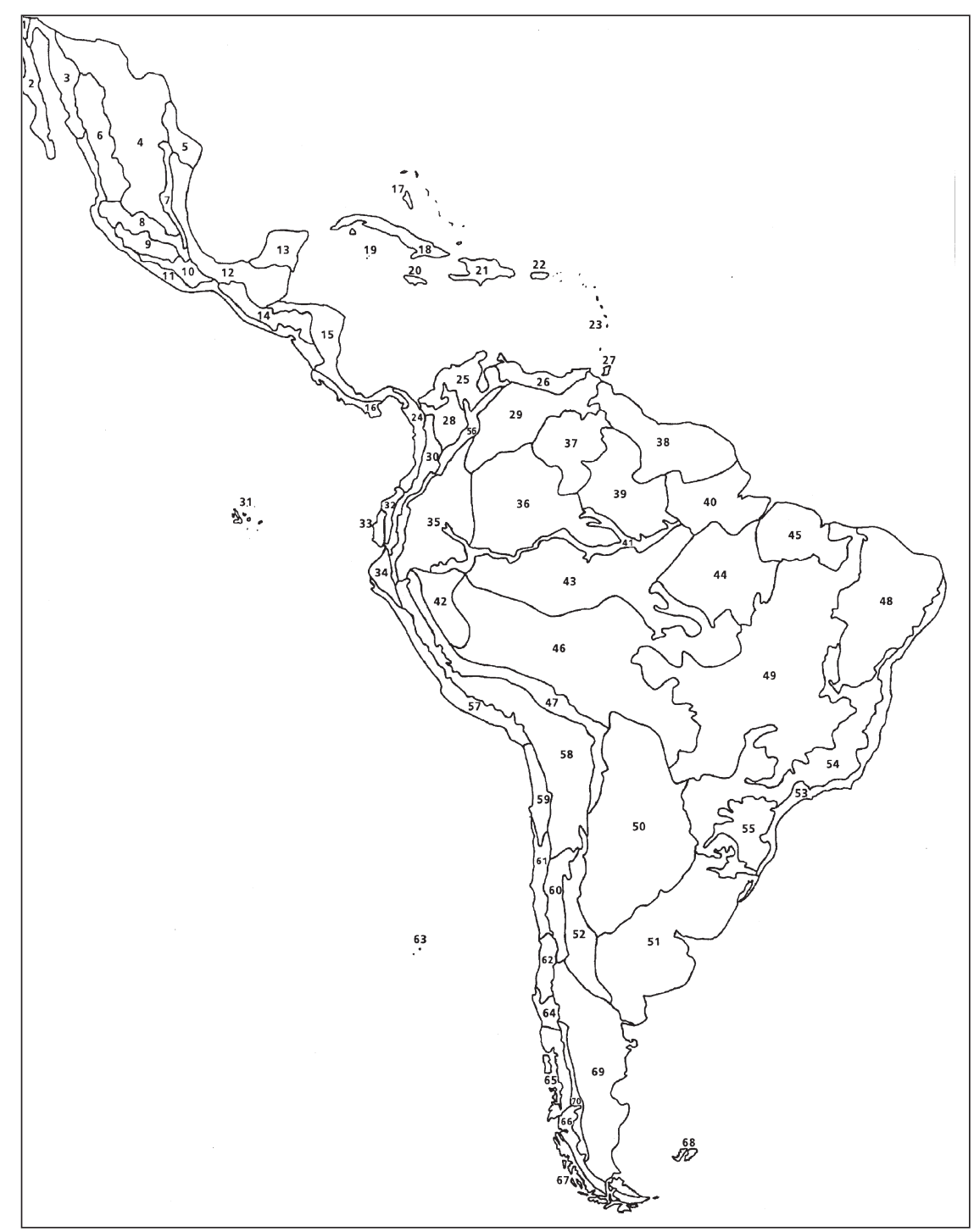

Fig. 11. Provincias biogeográficas de América Latina y el Caribe: 1, California; 2, Baja California; 3, Sonora; 4, Altiplano Mexicano; 5, Tamaulipas; 6, Sierra Madre Occidental; 7, Sierra Madre Oriental; 8, Eje Volcánico Transmexicano; 9, Cuenca del Balsas; 10, Sierra Madre del Sur; 11, Costa del Pacífico Mexicano; 12, Golfo de México; 13, Península de Yucatán; 14, Chiapas; 15, Este de América Central; 16, Oste del Istmo de Panamá; 17, Bahamas; 18, Cuba; 19, Islas Caimán; 20, Jamaica; 21, La Española; 22, Puerto Rico; 23, Antillas Menores; 24, Chocó; 25, Maracaibo; 26, Costa Venezolana; 27, Trinidad y Tobago; 28, Magdalena; 29, Llanos Venezolanos; 30, Cauca; 31, Islas Galápagos; 32, Oeste del Ecuador; 33, Ecuador Árido; 34, Tumbes-Piura; 35, Napo; 36, Imerí; 37, Guyana; 38, Guyana Húmeda; 39, Roraima; 40, Amapá; 41, Varzea; 42, Ucayali; 43, Madeira; 44, Tapajos-Xingu; 45, Pará; 46, Pantanal; 47, Yungas; 48, Caatinga; 49, Cerrado; 50, Chaco; 51, Pampa; 52, Monte; 53, Bosque Atlántico Brasileño; 54, Bosque Paranaense; 55; Bosque de Araucaria angustifolia; 56, Páramo Norandino; 57, Desierto Peruano Costero; 58, Puna; 59, Atacama; 60, Prepuna; 61, Coquimbo; 62, Santiago; 63, Islas Juan Fernández; 64, Maule; 65, Bosque Valdiviano; 66, Bosque Magallánico; 67, Páramo Magallánico; 68, Islas Malvinas; 69, Patagonia Central; 70, Patagonia Subandina.

Tehuantepec su límite de distribución austral, aunque pueden poseer algunas especies en América Central; se encuentran en bosques de coníferas y pastizales por encima de los $1700 \mathrm{~m}$ de altitud; y sus taxones más afines de encuentran en los Estados Unidos y Canadá.

Morrone \& MÁrquez (2001) llevaron a cabo un análisis panbiogeográfico de varias especies de Coleoptera de la zona de transición Mexicana, hallando dos trazos generalizados: uno septentrional, que comprende básicamente áreas montañosas de las Sierras Madre Occidental, Oriental y del
Sur, el Eje Volcánico Transmexicano y la Cuenca del Balsas; y otro meridional, que comprende la Sierra Madre de Chiapas y áreas bajas de Chiapas, el Golfo de México y la costa del Pacífico, llegando por el sur hasta el istmo de Panamá. Teniendo en cuenta también los resultados de otros análisis (MORRONE et al.1999; Espinosa et al.2000; Contreras-Medina \& EliosaLEÓN 2001), es posible reconocer cinco componentes bióticos para México (MORRONE \& MÁRQUEZ 2003), que reciben categoría de dominio. El dominio Neártico Californiano incluye la península de Baja California. El dominio Neártico Continental 


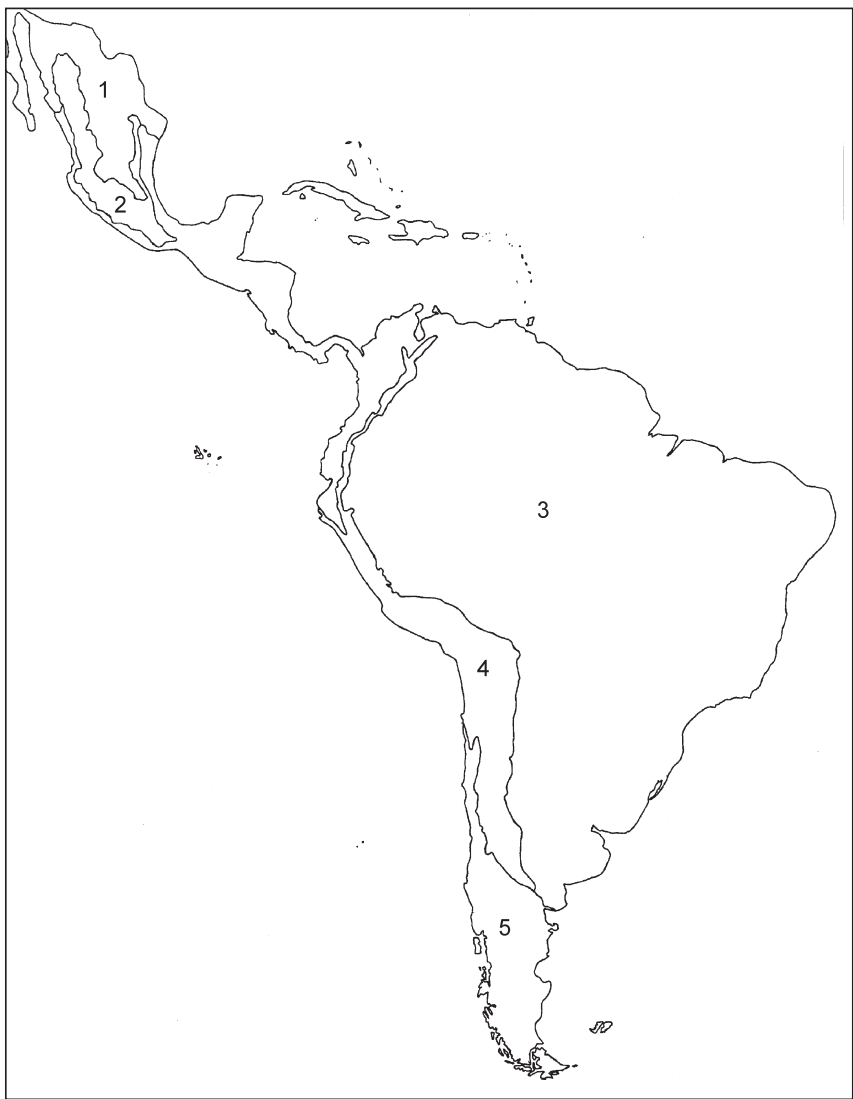

Fig. 12. Regiones y zonas de transición de América Latina y el Caribe: 1, región Neártica; 2, zona de transición Mexicana; 3, región Neotropical; 4, zona de transición Sudamericana; 5, región Andina.

se extiende por Sonora, el Altiplano Mexicano y Tamaulipas, extendiéndose hacia el norte hasta los Estados Unidos. El dominio Antillano se extiende por las Antillas, en México solo abarca la península de Yucatán. El dominio Mesoamericano abarca América Central, la Sierra Madre de Chiapas, y las tierras bajas del Golfo de México, la costa del océano Pacífico y América Central al norte del Lago Nicaragua. El dominio Mexicano de Montaña incluye las Sierras Madre Oriental, Occidental y del Sur, el Eje Volcánico Transmexicano y la Cuenca del Balsas.

Creo conveniente restringir la zona de transición Mexicana en sentido estricto al dominio Mexicano de Montaña, el cual posee cinco provincias: Sierra Madre Occidental (oeste de México, por encima de los $1000 \mathrm{~m}$ de altitud), Sierra Madre Oriental (este de México, por encima de los $1500 \mathrm{~m}$ de altitud), Eje Volcánico Transmexicano (centro de México), Cuenca del Balsas (centro de México, por debajo de los $2000 \mathrm{~m}$ de altitud, entre el Eje Volcánico Transmexicano y la Sierra Madre del Sur) y Sierra Madre del Sur (centro sur de México, por encima de los $1000 \mathrm{~m}$ de altitud).

Región Neotropical. Trópicos del Nuevo Mundo, en la mayor parte de América del Sur, toda América Central, el sur de México, las Antillas y el sur de la península de Florida. En tiempos precuaternarios la biota neotropical sudamericana se expandió hacia el norte, hasta América Central y México, y hacia el sur, a la región Andina y la zona de transición Sudamericana. Esta región se asigna al reino Holotropical, el cual incluye las áreas tropicales del mundo entre los $30^{\circ}$ de latitud sur y los $30^{\circ}$ de latitud norte, y que posee otras tres regiones: Etiópica o Afrotropical (África central, la península Arábiga, Madagascar y las islas del oeste del océano Índico), Oriental (India, Burma, Malasia, Indonesia, las Filipinas y las islas del Pacífico) y Australiana Tropical (noroeste de Australia). Corresponde a la Gondwana Oriental. Posee cuatro subregiones: Caribeña, Amazónica, Chaqueña y Paranaense.

La subregión Caribeña se extiende por el sur de México, América Central, las Antillas y el noroeste de América del Sur. Posee 24 provincias, las cuales pueden agruparse preliminarmente en tres dominios. El dominio Mesoamericano está integrado por cinco provincias: Costa del Pacífico Mexicano (franja estrecha de la costa del Pacífico de México), Golfo de México (costa del Golfo de México, en el este de México, Belice y el norte de Guatemala), Chiapas (sur de México, Guatemala, Honduras, El Salvador y Nicaragua; corresponde básicamente a la Sierra Madre de Chiapas, de 500 a $2000 \mathrm{~m}$ de altitud), Este de América Central (este de América Central, desde Guatemala hasta Panamá) y Oeste del Istmo de Panamá (oeste de América Central, desde Costa Rica hasta Panamá). El dominio Antillano está integrado por ocho provincias: Península de Yucatán (península de Yucatán), Bahamas (archipiélago de las Bahamas), Cuba (isla de Cuba), Islas Caimán (archipiélago que incluye las islas Grand Cayman, Little Cayman y Cayman Brac), Jamaica (isla de Jamaica), La Española (República Dominicana y Haití), Puerto Rico (isla de Puerto Rico) y Antillas Menores (archipiélagos de las Antillas Menores y de las Islas Vírgenes). El dominio del Noroeste de América del Sur está integrado por 11 provincias: Chocó (costa del Pacífico de Ecuador, Colombia y Panamá), Maracaibo (norte de Colombia y noroeste de Venezuela), Costa Venezolana (norte de Venezuela y Colombia), Trinidad y Tobago (islas de Trinidad y Tobago), Magdalena (oeste de Venezuela y noroeste de Colombia), Llanos Venezolanos (llanuras de gran parte de Venezuela y noroeste de Colombia), Cauca (oeste de Colombia y Ecuador), Islas Galápagos (archipiélago de Colón, en el océano Pacífico, $950 \mathrm{~km}$ al oeste de la costa de Ecuador), Oeste de Ecuador (oeste de Ecuador y sudoeste de Colombia), Ecuador Árido (oeste de Ecuador) y Tumbes-Piura (sur de Ecuador y norte del Perú).

La subregión Amazónica se extiende por Brasil, las Guyanas, Venezuela, Colombia, Ecuador, Perú, Bolivia, Paraguay y Argentina. Consta de 13 provincias: Napo (norte del Perú, sudoeste de Colombia y este de Ecuador), Imerí (sur de Venezuela, sudoeste de Colombia, norte del Perú y norte del Brasil), Guyana (noroeste de América del Sur, en el Escudo Guyano, entre Venezuela, Colombia, Guyana, Surinam y norte del Brasil), Guyana Húmeda (sudoeste de Venezuela, norte de Brasil, Surinam y Guyana), Roraima (norte del Brasil, sudoeste de Venezuela, Surinam y Guyana), Amapá (Surinam y noreste del Brasil), Varzea (noroeste del Brasil y del Perú), Ucayali 
(este del Perú, norte de Bolivia y oeste del Brasil), Madeira (noroeste del Brasil, limitando con los ríos Amazonas, Madeira, Beni y Xingu, y con la cordillera oriental de Bolivia), TapajosXingu (noroeste del Brasil), Pará (noroeste del Brasil, limitando con los ríos Tocantins, Araguaia, Grajau y Guana, y con la Serra do Gurupi), Pantanal (sur centro del Brasil, noroeste de Bolivia y norte del Paraguay) y Yungas (laderas occidentales de los Andes, entre los 300 y 3500 m de altitud, desde el norte del Perú hasta el noroeste de la Argentina).

La subregión Chaqueña se extiende por el norte y centro de la Argentina, sur de Bolivia, oeste y centro de Paraguay, Uruguay, y centro y noreste del Brasil. Se relaciona con las subregiones Amázonica y Paranaense; el desarrollo de un "corredor de sabana" durante el terciario actuó como el evento vicariante que separó en dos el bosque continuo. Consta de cuatro provincias: Caatinga (noroeste del Brasil), Cerrado (sur centro del Brasil, noreste del Paraguay y Bolivia), Chaco (sur de Bolivia, oeste del Paraguay, sur del Brasil y centro norte de la Argentina) y Pampa (centro oeste de la Argentina entre los 30 y $39^{\circ}$ de latitud sur, Uruguay y el sur de Rio Grande do Sul).

La subregión Paranaense se extiende por el noreste de la Argentina, este del Paraguay, y sur y este del Brasil. Aunque está más relacionada con la subregión Amazónica, algunos taxones muestran relaciones con la subregión Subantártica, señalando conexiones durante el terciario, cuando un clima templado permitió la existencia de un bosque continuo, el cual se extendió más al sur que en la actualidad, y que se fragmentó durante el oligoceno y mioceno, debido a la aridez ocasionada por el ascenso de los Andes y la expansión de la biota chaqueña. Posee tres provincias: Bosque Atlántico Brasileño (franja estrecha a lo largo de la costa del Brasil al este de la cordillera de la costa, entre los $7^{\circ}$ y $32^{\circ}$ de latitud sur), Bosque Paranaense (sur del Brasil, noreste de la Argentina y este del Paraguay) y Bosque de Araucaria angustifolia (sur del Brasil y noreste de la Argentina, entre 600 y $1800 \mathrm{~m}$ de altitud).

Zona de transición Sudamericana. Se extiende a lo largo de los Andes entre el oeste de Venezuela y el norte de Chile, y el centro oeste de la Argentina. Equivale a la subregión Páramo Puneña de Morrone (2001a). Dadas sus relaciones bióticas con elementos diferentes, las provincias incluidas aquí fueron asignadas anteriormente a las regiones Neotropical y Andina; el análisis de KaTINAS et al. (1999) ha demostrado claramente la naturaleza compuesta de esta área. Consta de seis provincias: Páramo Norandino (altas cordilleras de Venezuela, Colombia, Ecuador y Perú, por encima de los $3000 \mathrm{~m}$ de altitud), Desierto Peruano Costero (franja angosta a lo largo de la costa del Pacífico, desde el norte del Perú hasta el norte de Chile), Puna (Bolivia, norte de la Argentina y Chile, y sur del Perú), Atacama (norte de Chile, entre los 18 y $28^{\circ}$ de latitud sur), Prepuna (centro y noroeste de la Argentina) y Monte (centro de la Argentina, entre los 24 y $43^{\circ}$ de latitud sur).

Región Andina. Se extiende por el centro de Chile y la Patagonia. Se incluye en el reino Austral, el cual posee otras cinco regiones: Antártica (Antártida), Capense o Afrotemplada
(Sudáfrica), Neoguineana (Nuevas Guinea y Nueva Caledonia), Australiana Templada (sureste de Australia) y Neocelandesa (Nueva Zelanda). Corresponde a la Gondwana Occidental. Posee tres subregiones: Chilena Central, Subantártica y Patagónica.

La subregión Chilena Central se extiende por el centro de Chile, entre $\operatorname{los} 30^{\circ}$ y $34^{\circ}$ de latitud sur. Se relaciona con la subregión Subantártica, en especial con sus dos provincias más septentrionales (Maule y Bosque Valdiviano). Consta de dos provincias: Coquimbo (centro norte de Chile, entre los $28^{\circ}$ y $32^{\circ}$ de latitud sur) y Santiago (centro sur de Chile, entre los $32^{\circ}$ y $36^{\circ}$ de latitud sur).

La subregión Subantártica se extiende por los Andes australes, desde los $36^{\circ}$ de latitud sur hasta el Cabo de Hornos, incluyendo el archipiélago del sur de Chile y la Argentina, y las islas Malvinas, Georgia del Sur y Juan Fernández. Posee relaciones con la subregión Chilena Central y con áreas extraamericanas del reino Austral. Consta de seis provincias: Islas Juan Fernández (islas chilenas de Masatierra o Robinson Crusoe, Masafuera o Alejandro Selkirk, y Santa Clara, situadas en el océano Pacífico, $600 \mathrm{~km}$ al oeste de Valparaíso, a los $33^{\circ}$ de latitud sur), Maule (sur de Chile y la Argentina, entre los $36^{\circ}$ y $39^{\circ}$ de latitud sur), Bosque Valdiviano (sur de Chile y la Argentina, al sur de la provincia del Maule, alcanzando los $47^{\circ}$ de latitud sur), Bosque Magallánico (sur de Chile, desde los $47^{\circ}$ de latitud sur hasta el Cabo de Hornos y el sur de la Argentina en pequeñas partes del oeste de Santa Cruz y Tierra del Fuego), Páramo Magallánico (sur de Chile y la Argentina, desde el Golfo de Penas, a los $48^{\circ}$ de latitud sur, hasta el Cabo de Hornos, limitando al oeste con el Bosque Magallánico) e Islas Malvinas (archipiélago argentino situado en el océano Atlántico Sur, a 550 km de Tierra del Fuego).

La subregión Patagónica se extiende en el sur de la Argentina, alcanzando Chile en algunas partes de Aisén y Magallanes. Consta de dos provincias: Patagonia Central (sur de la Argentina y Chile) y Patagonia Subandina (sudoeste de la Argentina, constituye una franja estrecha a los largo de los Andes australes, al sur de los $51^{\circ}$ de latitud sur).

\section{DISCUSIÓN}

Morrone (2001g) discutió los conceptos de homología biogeográfica primaria y secundaria, incluyendo bajo la primera el reconocimiento de componentes bióticos, a través del enfoque panbiogeográfico y del análisis de parsimonia de endemismos. Otros autores han reconocido conceptos semejantes, como líneas filéticas (JEAnNel 1942), linajes (Ringuelet 1961), cenocrones (Reig 1962, 1981), corotipos (BARONI-URBANi et al.1978), patrones de distribución (HALFFTER 1978, 1987, 2003), componentes (CADLE 1985) y elementos bióticos (HAUSDORF 2002); faltaría un análisis comparativo con el fin de establecer con precisión cuánto difieren entre sí. Recientemente, Hausdorf (2002; ver también HAUSDORF \& HeNNIG [2003]) ha destacado la importancia de considerar "elementos bióticos" como unidades biogeográficas, en lugar de las áreas de endemismo que se emplean en los análisis 
biogeográficos cladísticos. Estos elementos o componentes bióticos resultan básicamente de procesos vicariantes, pero a diferencia de las áreas de endemismo pueden reconocerse aun cuando haya habido eventos de dispersión o expansiones de las distribuciones de algunos taxones particulares. El enfoque panbiogeográfico ha mostrado ser particularmente útil para reconocerlos, como lo demuestra la cantidad de análisis empíricos llevados a cabo en las últimas décadas.

Las zonas de transición, localizadas en los límites entre regiones biogeográficas, representan eventos de "hibridación" biótica, promovidos por cambios ecológicos que permiten la mezcla de componentes bióticos diferentes. A pesar de que pueden poseer una biota empobrecida (DARLINGTON 1957), en ocasiones exhiben una diversidad inusualmente alta, como es el caso de la zona de transición Mexicana. Desde un punto de vista evolutivo, las zonas de transición merecen atención especial, pues más que líneas estáticas, los límites entre regiones son áreas de interacción biótica intensa (RUGGIERO \& Ezcurra 2003). Por ello, el reconocimiento de zonas de transición y la identificación de los diferentes componentes bióticos presentes en ellas son de gran relevancia. Aquí también la panbiogeografía posee gran utilidad, pues la localización de nodos en ciertas áreas es una manera de identificar zonas de transición.

Agradecimientos. Agradezco a dos árbitros anónimos por sus valiosas sugerencias.

\section{REFERENCIAS}

Aguilar Aguilar, R. \& R. Contreras Medina. 2001. La distribución de los mamíferos de México: Un enfoque panbiogeográfico, p. 213-219. In: J. Llorente Bousquets \& J. J. Morrone (eds.). Introducción a la biogeografía en Latinoamérica: Teorías, conceptos, métodos y aplicaciones. México, Las Prensas de Ciencias, Facultad de Ciencias, UNAM.

Andersson, L. 1996. An ontological dilema: Epistemology and methodology of historical biogeography. Journal of Biogeography 23: 269-277.

Baroni-Urbani, C.; S. Rufo \& A. Vigna. 1978. Materiali per una biogeografía italiana fondata su alcuni generi di coleotteri cicindelidi, carabidi e crisomelidi. Estrati e Memoria della Societá Entomologica Italiana 56: 35-92.

Bellan, G. \& D. Bellan Santini. 1997. Utilizzazione delle analisi di parsimonia (cladistica) in sinecologia bentonica: Esempi in una zona inquinata. Societá Italiana di Ecologia Atti 18: 247-250.

Bisconti, M.; W. Landini; G. Bianucci; G. Cantalamessa; G. Carnevale; L. Ragaini \& G. VAlleri. 2001. Biogeographic relationships of the Galapagos terrestrial biota: Parsimony analyses of endemicity based on reptiles, land birds and Scalesia land plants. Journal of Biogeography 28: 495-510.

Cadle, J. E. 1985. The Neotropical colubrid snake fauna (Serpentes: Colubridae): Lineage components and biogeography. Systematic Zoology 34: 1-20.

Carvalho, C. J. B. de; M. Bortolanza; M. C. Cardoso da Silva \& E. D. Giustina Soares. 2003. Distributional patterns of the Neotropical Muscidae (Diptera), p. 263-274. In: J. J. Morrone \& J. Llorente Bousquets (eds.). Una perspectiva latinoamericana de la biogeografía. México, Las Prensas de Ciencias, Facultad de Ciencias, UNAM.
Colacino, C. 1997. Léon Croizat's biogeography and macroevolution, or... "out of nothing, nothing comes". Philippine Scientist 34: $73-88$.

Contreras Medina, R. \& H. Eliosa León. 2001. Una visión panbiogeográfica preliminar de México, p. 197-211. In: J. LLORENTE Bousquets \& J. J. Morrone (eds.). Introducción a la biogeografía en Latinoamérica: Conceptos, teorías, métodos y aplicaciones. México, Las Prensas de Ciencias, UNAM.

Contreras Medina, R.; I. Luna Vega \& J. J. Morrone. 1999. Biogeographic analysis of the genera of Cycadales and Coniferales (Gymnospermae): A panbiogeographic approach. Biogeographica 75: $163-176$.

Cortés B. R. \& P. Franco R. 1997. Análisis panbiogeográfico de la flora de Chiribiquete, Colombia. Caldasia 19: 465-478.

Cox, C. B. 1998. From generalised tracks to ocean basins- How useful is biogeography? Journal of Biogeography 25: 813-828.

Cracraft, J. 1991. Patterns of diversification within continental biotas: Hierarchical congruence among the areas of endemism of Australian vertebrates. Australian Systematic Botany 4: 211-227.

Craw, R. C. 1988. Continuing the synthesis between panbiogeography, phylogenetic systematics and geology as illustrated by empirical studies on the biogeography of New Zealand and the Chatham Islands. Systematic Zoology 37: 291-310.

Craw, R. C. 1989a. New Zealand biogeography: A panbiogeographic approach. New Zealand Journal of Zoology 16: 527-547.

Craw, R. C. 1989b. Quantitative panbiogeography: Introduction to methods. New Zealand Journal of Zoology 16: 485-494.

Craw, R. C.; J. R. Grehan \& M. J. Heads. 1999. Panbiogeography: Tracking the history of life. New York y Oxford, Oxford Biogeography series 11 .

Craw, R. C. \& R. Page. 1988. Panbiogeography: Method and metaphor in the new biogeography, p. 163-189. In: M.-W. Ho \& S. W. Fox (eds). Evolutionary processes and metaphors. Chichester, John Wiley and Sons.

Craw, R. C. \& P. Weston. 1984. Panbiogeography: A progressive research program? Systematic Zoology 33: 1-33.

Crisci, J. V. 2001. The voice of historical biogeography. Journal of Biogeography 28: 157-168.

Crisci, J. V.; S. Freire; G. Sancho \& L. Katinas. 2001. Historical biogeography of the Asteraceae from Tandilia and Ventania mountain ranges (Buenos Aires, Argentina). Caldasia 23: 21-41.

CRisCi, J. V.; L. Katinas \& P. Posadas. 2000. Introducción a la teoría y práctica de la biogeografía histórica. Buenos Aires, Sociedad Argentina de Botánica. (Traducción al inglés: 2003, Historical biogeography: An introduction. Cambridge, Massachussets y Londres, Harvard University Press)

Croizat, L. 1958. Panbiogeography. Vols. 1 y 2. Caracas, Publicado por el autor.

Croizat, L. 1964. Space, time, form: The biological synthesis. Caracas, Publicado por el autor.

Croizat, L.; G. Nelson \& D. E. Rosen. 1974. Centers of origin and related concepts. Systematic Zoology 23: 265-287.

Darlington, P. J. 1957. Zoogeography: The geographical distribution of animals. New York, John Wiley and Sons.

DARWIN, C. R. 1859. The origin of species by means of natural selection or the preservation of favoured races in the struggle for life. London, John Murray.

Da Silva, J. M. C. \& D. C. Oren. 1996. Application of parsimony analysis of endemicity in Amazonian biogeography: An example with primates. Biological Journal of the Linnean Society 39: 427-437.

Donoghue, M. J.; C. D. Bell \& J. Li. 2001. Phylogenetic patterns in northern hemisphere plant geography. Internacional Journal of Plant Science 162: S41-S52.

Escalante, T. \& J. J. Morrone. 2003. ¿Para qué sirve el análisis de parsimonia de endemismos?, p. 167-172. In: J. J. Morrone \& J. Llorente Bousquets (eds.). Una perspectiva latinoamericana de la biogeografía. México, Las Prensas de Ciencias, Facultad de Ciencias, UNAM.

Espinosa Organista, D.; J. J. Morrone; C. Aguilar \& J. Llorente Bousquets. 
2000. Regionalización biogeográfica de México: Provincias bióticas, p. 61-94. In: J. Llorente Bousquets; E. GonzÁlez \& N. Papavero (eds.). Biodiversidad, taxonomía y biogeografía de artrópodos de México: Hacia una síntesis de su conocimiento. Vol. II. México, Conabio.

Fernandes, M. E. B.; J. M. Da Silva \& J. Silva Jr. 1995. The monkeys of the islands of the Amazon estuary, Brazil: A biogeographic analysis. Mammalia 59: 213-221.

Franco Rosselli, P. \& C. C. Berg. 1997. Distributional patterns of Cecropia (Cecropiaceae): A panbiogeographic analysis. Caldasia 19: 285-296.

García Barros, E.; P. Gurrea; M. J. Luciáñez; J. M. Cano; M. L. Munguira; J. C. Moreno; H. Sainz; M. J. Sanza \& J. C. Simón. 2002. Parsimony analysis of endemicity and its application to animal and plant geographical distributions in the Ibero-Balearic region (western Mediterranean). Journal of Biogeography 29: 109-124.

GeraAds, D. 1998. Biogeography of circum-Mediterranean MiocenePliocene rodents: A revision using factor analysis and parsimony analysis of endemicity. Palaeogeography, Palaeoclimatology, Palaeoecology 137: 273-288.

Glasby, C. J. \& B. Álvarez. 1999. Distribution patterns and biogeographic analysis of Austral Polychaeta (Annelida). Journal of Biogeography 26: 507-533.

Grande, L. 1990. Vicariance biogeography, p. 448-451. In: D. E. G. Briggs \& P. R. Crowther (eds.). Paleobiology: A synthesis. Oxford, Blackwell Scientific Publications.

Gray, R. D. 1988. Metaphors and methods: Behavioral ecology, panbiogeography and the evolving synthesis, p. 209-242. In: M.W. Ho \& S. Fox (eds.). Evolutionary processes and metaphors. Chichester, John Wiley and Sons.

Gray, R. D. 1989. Oppositions in panbiogeography: Can the conflict between selection, constraint, ecology, and history be resolved? New Zealand Journal of Zoology 16: 787-806.

Gray, R. D. 1992. Death of the gene: Developmental systems strike again, p. 165-209. In: P. Griffiths (ed.). Trees of life: Essays in philosophy of biology. Boston, Kluwer Academic Publishers.

GREHAN, J. R. 1984. Evolution by law: Croizat's 'orthogeny' and Darwin's 'laws of growth'. Tuatara 27: 14-19.

Grehan, J. R. 1988a. Panbiogeography: Evolution in space and time. Rivista di Biologia, Biological Forum 81: 469-498.

Grehan, J. R. 1988b. Biogeographic homology: Ratites and the southern beeches. Rivista di Biologia, Biological Forum 81: 577-587.

Grehan, J. R. 1991. Panbiogeography 1981-91: Development of an earth/life synthesis. Progress in Physical Geography 15: 331 363.

Grehan, J. R. 1994. The beginning and end of dispersal: The representation of 'panbiogeography'. Journal of Biogeography 21: 451-462.

GrehAN, J. R. 2001a. Islas Galápagos: Biogeografía, tectónica y evolución en un archipiélago oceánico, p. 153-160. In: J. LloRENTE BousQuets \& J. J. Morrone (eds.). Introducción a la biogeografía en Latinoamérica: Teorías, conceptos, métodos y aplicaciones. México, Las Prensas de Ciencias, Facultad de Ciencias, UNAM.

Grehan, J. R. 2001b. Panbiogeografía y la geografía de la vida, p. 181195. In: J. Llorente Bousquets \& J. J. Morrone (eds.). Introducción a la biogeografía en Latinoamérica: Teorías, conceptos, métodos y aplicaciones. México, Las Prensas de Ciencias, Facultad de Ciencias, UNAM

Grehan, J. R. 2001c. Biogeography and evolution of the Galapagos: Integration of the biological and geological evidence. Biological Journal of the Linnean Society 74: 267-287.

Grehan, J. R. 2001d. Panbiogeography from tracks to ocean basins: Evolving perspectives. Journal of Biogeography 28: 413-429.

Grehan, J. R. \& R. Ainsworth. 1985. Orthogenesis and evolution. Systematic Zoology 34: 174-192.

Grene, M. 1990. Is evolution at crossroads? Evolutionary Biology 24: $51-81$.

HaLffter, G. 1978. Un nuevo patrón de dispersión en la zona de transición mexicana: El mesoamericano de montaña. Folia Entomológica Mexicana 39-40: 219-222.
HalfFter, G. 1987. Biogeography of the montane entomofauna of Mexico and Central America. Annual Review of Entomology 32: $95-$ 114.

Halffter, G. 2003. Biogeografía de la entomofauna de montaña de México y América Central, p. 87-97. In: J. J. Morrone \& J. Llorente Bousquets (eds.). Una perspectiva latinoamericana de la biogeografía. México, Las Prensas de Ciencias, Facultad de Ciencias, UNAM.

Hausdorf, B. 2002. Units in biogeography. Systematic Biology 51: 648-652.

Hausdorf, B. \& C. Hennig. 2003. Biotic element analysis in biogeography. Systematic Biology 52: 717-723.

Haydon, D. T.; B. I. Crother \& E. R. Pianka. 1994. New directions on biogeography? Trends in Ecology and Evolution 10: 403-406.

Heads, M. J. 1985. On the nature of ancestors. Systematic Zoology 34: $205-215$.

Heads, M. J. 1986. A panbiogeographic analysis of Auckland islands archipelago, p. 30-44. In: R. D. ARChiBALD (ed.). The Lepidoptera, bryophytes, and panbiogeography of Auckland islands. Dunedin, New Zealand Entomological Society.

Heads, M. J. 1989. Integrating earth and life sciences in New Zealand natural history: The parallel arcs model. New Zealand Journal of Zoology 16: 549-585.

Henderson, I. M. 1989. Quantitative panbiogeography: An investigation into concepts and methods. New Zealand Journal of Zoology 16: $495-510$

Henderson, I. M. 1991. Biogeography without area? Australian Systematic Botany 4: 59-71.

Holloway, J. D. 2003. Biological images of geological history: Through a glass darkly or brightly face to face. Journal of Biogeography 30: $165-179$.

Hooker, J. D. 1844-60. The botany of the Antarctic Voyage of $\mathbf{H}$. M. Discovery ships Erebus and Terror in the years 18391843. I. Flora Antarctica (1844-47). London.

Humphries, C. J. \& L. R. Parenti. 1999. Cladistic biogeographySecond edition: Interpreting patterns of plant and animal distributions. Oxford, Oxford University Press.

Humphries, C. J. \& O. Seberg. 1989. Graphs and generalized tracks: Some comments on method. Systematic Zoology 38: 69-76.

IpPI, S. \& V. Flores. 2001. Las tortugas neotropicales y sus áreas de endemismo. Acta Zoológica Mexicana (nueva serie) 84: 49-63.

Jeannel, R. 1942. Le génese des faunes terrestres. Paris, Press Universitaires de France.

Katinas, L.; J. J. Morrone \& J. V. Crisci. 1999. Track analysis reveals the composite nature of the Andean biota. Australian Systematic Botany 47: 111-130.

Linder, H. P. \& D. M. MANN. 1998. The phylogeny and biogeography of Thamnochortus (Restionaceae). Botanical Journal of the Linnean Society 128: 319-357.

Lopretto, E. C. \& J. J. Morrone. 1998. Anaspidacea, Bathynellacea (Syncarida), generalised tracks, and the biogeographical relationships of South America. Zoologica Scripta 27: 311-318.

LOURENÇO, W. R. 1998. Panbiogeographie, les distributions disjointes et le concept de famille relictuelle chez les scorpions. Biogeographica 74: 133-144.

Luna Vega, I.; O. Alcántara, D. Espinosa Organista \& J. J. Morrone. 1999. Historical relationships of the Mexican cloud forests: A preliminary vicariance model applying parsimony analysis of endemicity to vascular plant taxa. Journal of Biogeography 26 : 1299-1305.

Luna Vega, I.; O. Alcántara, J. J. Morrone \& D. Espinosa Organista. 2000. Track analysis and conservation priorities in the cloud forests of Hidalgo, Mexico. Diversity and Distributions 6: 137-143.

Luna Vega, I. \& R. Contreras-Medina. 2000. Distribution of the genera of Theaceae (Angiospermae: Theales): A panbiogeographic analysis. Biogeographica 76: 79-88.

Luna Vega, I.; J. J. Morrone, O. Alcántara Ayala \& D. Espinosa Organista. 2001. Biogeographical affinities among Neotropical cloud forests. Plant Systematics and Evolution 228: 229-239.

Marino, P. I.; G. R. Sinelli \& P. Posadas. 2001. Distributional patterns 
of species of Ceratopogonidae (Diptera) in southern South America. Biogeographica 77: 113-122.

Matthew, W. D. 1915. Climate and evolution. Annals of the New York Academy of Sciences 24: 171-318.

MAYR, E. 1982. The growth of biological thought: Diversity, evolution, and inheritance. Cambridge, Massachusetts y London, Harvard University Press.

Menu Marque, S.; J. J. Morrone \& C. Locascio. 2000. Distributional patterns of the South American species of Boeckella (Copepoda: Centropagidae): A track analysis. Journal of Crustacean Biology 20: $262-272$.

Morrone, J. J. 1992. Revisión sistemática, análisis cladístico y biogeografía histórica de los géneros Falklandius Enderlein y Lanteriella gen. nov. (Coleoptera: Curculionidae). Acta Entomológica Chilena 17: 157-174.

Morrone, J. J. 1993a. Beyond binary oppositions. Cladistics 9: 437438.

Morrone, J. J. 1993b. Revisión sistemática de un nuevo género de Rhytirrhinini (Coleoptera: Curculionidae), con un análisis biogeográfico del dominio Subantártico. Boletín de la Sociedad de Biología de Concepción 64: 121-145.

Morrone, J. J. 1994a. Systematics, cladistics, and biogeography of the Andean weevil genera Macrostyphlus, Adioristidius, Puranius, and Amathynetoides, new genus (Coleoptera: Curculionidae). American Museum Novitates 3104: 1-63.

Morrone, J. J. 1994b. Distributional patterns of species of Rhytirrhinini (Coleoptera: Curculionidae) and the historical relationships of the Andean provinces. Global Ecology and Biogeography Letters 4: $188-194$.

Morrone, J. J. 1994c. On the identification of areas of endemism. Systematic Biology 43: 438-441.

Morrone, J. J. 1996. Austral biogeography and relict weevil taxa (Coleoptera: Nemonychidae, Belidae, Brentidae, and Caridae). Journal of Comparative Biology 1: 123-127.

Morrone, J. J. 1998. On Udvardy's Insulantarctica province: A test from the weevils (Coleoptera: Curculionoidea). Journal of Biogeography 25: 947-955.

Morrone, J. J. 2000a. What is the Chacoan subregion? Neotropica 46: 51-68.

Morrone, J. J. 2000b. A new regional biogeography of the Amazonian subregion, mainly based on animal taxa. Anales del Instituto de Biología de la UNAM, serie Zoología, 71: 99-123.

Morrone, J. J. 2000c. El tiempo de Darwin y el espacio de Croizat: Rupturas epistémicas en los estudios evolutivos. Ciencia 51: 3946.

Morrone, J. J. 2001a. Biogeografía de América Latina y el Caribe. Zaragoza, Manuales y Tesis SEA, nro. 3.

Morrone, J. J. 2001b. Toward a cladistic model for the Caribbean subregion: Delimitation of areas of endemism. Caldasia 23: 4376.

Morrone, J. J. 2001c. A formal definition of the Paramo-Punan biogeographic subregion and its provinces, based mainly on animal taxa. Revista del Museo Argentino de Ciencias Naturales, nueva serie, 3: 1-12.

Morrone, J. J. 2001d. A proposal concerning formal definitions of the Neotropical and Andean regions. Biogeographica 77: 65-82.

Morrone, J. J. 2001e. Review of the biogeographic provinces of the Patagonian subregion. Revista de la Sociedad Entomológica Argentina 60: 1-8.

Morrone, J. J. 2001f. The Parana subregion and its provinces. Physis (Buenos Aires) 58: 1-7.

Morrone, J. J. 2001g. Homology, biogeography and areas of endemism. Diversity and Distributions 7: 297-300.

Morrone, J. J. 2002. El espectro del dispersalismo: De los centros de origen a las áreas ancestrales. Revista de la Sociedad Entomológica Argentina 61(3-4): 1-14.

Morrone, J. J. \& J. M. Carpenter. 1994. In search of a method for cladistic biogeography: An empirical comparison of component analysis, Brooks parsimony analysis, and three area statements. Cladistics 10: 99-153.
Morrone, J. J. \& M. del C. Coscarón. 1996. Distributional patterns of the American Peiratinae (Heteroptera: Reduviidae). Zoologische Medelingen Leiden 70: 1-15.

Morrone, J. J. \& J. V. Crisci. 1990. Panbiogeografía: Fundamentos y métodos. Evolución Biológica 4: 119-140.

Morrone, J. J. \& J. V. CrisCi. 1995. Historical biogeography: Introduction to methods. Annual Review of Ecology and Systematics 26: 373-401.

Morrone, J. J.; D. Espinosa Organista; C. Aguilar-Zúñiga \& J. Llorente Bousquets. 1999. Preliminary classification of the Mexican biogeographic provinces: A parsimony analysis of endemicity based on plant, insect, and bird taxa. Southwestern Naturalist 44: 508-515.

Morrone, J. J.; D. Espinosa Organista \& J. Llorente Bousquets. 1996. Manual de biogeografía histórica. México, Universidad Nacional Autónoma de México.

Morrone, J. J.; L. Katinas \& J. V. CRisci. 1997. A cladistic biogeographic analysis of Central Chile. Journal of Comparative Biology 2: $25-42$

Morrone, J. J. \& E. C. Lopretto. 1994. Distributional patterns of freshwater Decapoda (Crustacea: Malacostraca) in southern South America: A panbiogeographic approach. Journal of Biogeography 21: $97-109$

Morrone, J. J. \& E. C. Lopretto. 1995. Parsimony analysis of endemicity of freshwater Decapoda (Crustacea: Malacostraca) from southern South America. Neotropica 41: 3-8.

Morrone, J. J. \& J. MÁrquez. 2001. Halffter's Mexican Transition Zone, beetle generalised tracks, and geographical homology. Journal of Biogeography 28: 635-650.

Morrone, J. J. \& J. Márquez. 2003. Aproximación a un Atlas Biogeográfico Mexicano: Componentes bióticos principales y provincias biogeográficas, p. 217-220. In: J. J. Morrone, \& J. Llorente Bousquets (eds.). Una perspectiva latinoamericana de la biogeografía. México, Las Prensas de Ciencias, Facultad de Ciencias, UNAM.

Morrone, J. J. \& L. A. Pereira. 1999. On the geographical distribution of the Neotropical and Andean species of Schendylops (Chilopoda: Geophilomorpha: Schendylidae). Revista de la Sociedad Entomológica Argentina 58: 165-171.

Myers, A. A. 1991. How did Hawaii accumulate its biota?: A test from the Amphipoda. Global Ecology and Biogeography Letters 1: 24-29.

Nelson, G. 1978. From Candolle to Croizat: Comments on the history of biogeography. Journal of the History of Biology 11: 269305 .

Nelson, G. 1985. A decade of challenge the future of biogeography. Journal of the History of Earth Sciences Society 4: 187-196.

Nelson, G. 1989. Cladistics and evolutionary models. Cladistics 5: 275-289.

Nelson, G. \& N. I. Platnick. 1981. Systematics and biogeography: Cladistics and vicariance. New York, Columbia University Press.

Orama, S. 2000. Evolution's eye: A systems view of the biologyculture divide. Durham y London, Duke University Press.

PAGE, R. D. M. 1987. Graphs and generalized tracks: Quantifying Croizat's panbiogeography. Systematic Zoology 36: 1-17.

Page, R. D. M. 1990. Tracks and trees in the Antipodes. Systematic Zoology 39: 288-299.

Patterson, C. 1981. Methods of paleobiogeography, p. 446-489. In: D. E. Rosen \& G. Nelson (eds.). Vicariance biogeography: A critique. New York, Columbia University Press.

Pinna, M. C. C., DE. 1991. Concepts and tests of homology in the cladistic paradigm. Cladistics 7: 367-394.

Platnick, N. I. \& G. Nelson. 1978. A method of analysis for historical biogeography. Systematic Zoology 27: 1-16.

Platnick, N. I. \& G. Nelson. 1988. Spanning-tree biogeography: Shortcut, detour, or dead-end? Systematic Zoology 37: 410-419.

Posadas, P. 1996. Distributional patterns of vascular plants in Tierra del Fuego: A study applying Parsimony Analysis of Endemicity (PAE). Biogeographica 72: 161-177.

Posadas, P. E.; J. M. Estévez \& J. J. Morrone. 1997. Distributional 
patterns and endemism areas of vascular plants in the Andean subregion. Fontqueria 48: 1-10.

Posadas, P. \& D. R. Miranda-Esquivel. 1999. El PAE (Parsimony Analysis of Endemicity) como una herramienta en la evaluación de la biodiversidad. Revista Chilena de Historia Natural 72: 539546.

ReIG, O. A. 1962. Las interacciones cenogenéticas en el desarrollo de la fauna de vertebrados tetrápodos de América del Sur. Ameghiniana 1: $131-140$.

Reig, O. A. 1981. Teoría del origen y desarrollo de la fauna de mamíferos de América del Sur. Mar del Plata, Museo Municipal de Ciencias Naturales Lorenzo Scaglia.

Ringuelet, R. A. 1961. Rasgos fundamentales de la zoogeografía de la Argentina. Physis (Buenos Aires) 22: 151-170.

Roig Juñent, S.; G. Flores \& C. Mattoni. 2003. Consideraciones biogeográficas de la Precordillera (Argentina), con base en artrópodos epígeos, p. 275-288. In: J. J. Morrone \& J. Llorente Bousquets (eds.). Una perspectiva latinoamericana de la biogeografía. México, Las Prensas de Ciencias, Facultad de Ciencias, UNAM.

Rojas Soto, O. R.; O. Alcántara Ayala \& A. G. Navarro. 2003. Regionalization of the avifauna of the Baja California peninsula, Mexico: A parsimony analysis of endemicity and distributional modelling approach. Journal of Biogeography 30: 449-461.

Ron, S. R. 2000. Biogeographic area relationships of lowland Neotropical rainforest based on raw distributions of vertebrate groups. Biological Journal of the Linnean Society 71: 379-402.

Rosen, B. R. 1988. From fossils to earth history: Applied historical biogeography, p. 437-481. In: A. A. Myers \& P. S. Giller (eds.). Analytical biogeography. London, Chapman and Hall.

Rosen, D. E. 1976. A vicariance model of Caribbean biogeography. Systematic Zoology 24: 431-464.

Ruggiero, A. \& C. Ezcurra. 2003. Regiones y transiciones biogeográficas: Complementariedad de los análisis en biogeografía histórica y ecológica, p. 141-154. In: J. J. Morrone, J. J. \& J. Llorente Bousquets (eds.). Una perspectiva latinoamericana de la biogeografía. México, Las Prensas de Ciencias, Facultad de Ciencias, UNAM.

SFENTHOURAKIS, S. \& S. GiokAs. 1998. A biogeographical analysis of Greek Oniscidean endemism. Israel Journal of Zoology 44: 273-282.

Simpson, G. G. 1965. The geography of evolution. Philadelphia y New York, Chilton,
Sмiтн, C. H. 1989. Historical biogeography: Geography as evolution, evolution as geography. New Zealand Journal of Zoology 16: 773-785.

Stace, C. A. 1989. Dispersal versus vicariance-no contest. Journal of Biogeography 16: 201-202.

Swofford, D. L. 1999. PAUP*. Phylogenetic analysis using parsimony (*and other methods). Version 4. Sunderland, Sinauer Associates.

Trejo Torres, J. C. 2003. Biogeografía ecológica de las Antillas: Ejemplos de las orquídeas y las selvas cársticas, p. 199-208. In: J. J. Morrone \& J. Llorente Bousquets (eds.). Una perspectiva latinoamericana de la biogeografía. México, Las Prensas de Ciencias, Facultad de Ciencias, UNAM.

Trejo Torres, J. C. \& J. D. Ackerman. 2001. Biogeography of the Antilles based on a parsimony analysis of orchid distributions. Journal of Biogeography 28: 775-794.

Trejo Torres, J. C. \& J. D. Ackerman. 2002. Composition patterns of Caribbean limestone forests: Are parsimony, classification, and ordination analyses congruent? Biotropica 34: 502-515.

VARGAS, J. M. 1992a. Un ensayo en torno al concepto de biogeografía. Monografías en Herpetología 2: 7-20.

VARGAS, J. M. 1992b. Escuelas y tendencias en biogeografía histórica. Monografías en Herpetología 2: 107-136.

Vargas, J. M. 2002. Proyecto docente de zoogeografía. Málaga, Presentación para concurso de plaza de Catedrático, Universidad de Málaga.

Vuilleumier, E. 1999. Biogeography on the eve of the twenty-first century: Towards an epistemology of biogeography. Ostrich 70: 89-103.

Wallace, A. R. 1876. The geographical distribution of animals, with a study of the relations of living and extinct faunas as elucidating the past changes of the Earth's surface. Vol. I. London, Macmillan and Company.

Watanabe, K. 1998. Parsimony analysis of the distribution patterns of Japanese primary freshwater fishes, and its application to the distribution of the bagrid catfishes. Ichthyological Research 45: 259-270.

Zunino, M. \& A. Zullini. 1995. Biogeografia: La dimensione spaziale dell'evoluzione. Milán, Casa Editrice Ambrosiana. (Traducción al español: 2003, Biogeografía: La dimensión espacial de la evolución. México, D. F., Fondo de Cultura Económica).

Recibido en 13.I.2004; aceptado en 30.IV.2004 